\title{
Graphical User Interface (GUI) for Local Positioning System Based on Labview
}

\author{
Hameedah Sahib Hasan, Mohamed Hussein, Shaharil Mad Saad, and Mohd Azuwan Mat Dzahir
}

\begin{abstract}
Local position system (LPS) becomes more and more diversified and increasingly important in large numbers of applications and contexts such as healthcare, targeted, monitoring, tracking and security. In LPS, three anchors at least with known position are employed with one tag for localization. In this paper, LPS which contains three anchors and one tag is used to get positioning. Tag position represents user location. It estimates by using UWB technology which sending signal based on triangulation method with assist of Labview. By using Labview coding the position of each anchors can be read successful after connection all anchors and tag with $P C$ and router. The positioning of tag in $(x, y)$ direction, successfully update immediately when tag moving which is shown in graph. Therefore, the Graphical User Interface (GUI) for LPS based on Labview is achieved to determine the user positioning with positioning error less than $17 \mathrm{~cm}$. We believe this paper would catalyze further investigation by the researcher which deal with both of LPS field and Labview.
\end{abstract}

Index Terms-Local positioning system, anchors, tag, UWB, graphical user interface, Labview.

\section{INTRODUCTION}

LPS is a technique used to obtain the position of people or objects inside a building that is covered by a local area network [1]. The use of LPS has become a true necessary for which a various technology have considered in order to estimate user's position with a good accuracy. There are many types of technologies used in LPS such as infrared radiation (IR), radio frequency identification (RFID), Bluetooth, Wi-Fi, ultrasound, vision-based systems and ultra-wideband (UWB) [2], [3]. LPS is attracted scientific and enterprise interest because it has many opportunity for applying these technologies in different aspects such as hospital, building, market, and shopping mall [4]. For the last a few years, there are many researchers have been done in LPS, however, most of them deal with performance parameter such as accuracy, cost, power consumption, etc. In addition LPS has many challenges in term of accuracy from either accuracy or coverage limitation [2], [5], [6]. LPS uses maps in real time to assist people in navigation task, which is known commercial applications [1]. LPS also is used for

Manuscript received August 15, 2018; revised February 19, 2019.

Hameedah Sahib Hasan is with Ministry of Higher Education and Scientific Research, Al-Furat Al-Awsat Technical University, Iraq (e-mail: hameedah211ou@gmail.com).

Mohamed Hussein, Shaharil Mad Saad, and Mohd Azuwan Mat Dzahir are with Faculty of Mechanical Engineering, Universiti Teknologi Malaysia (UTM), Johor Bauru, 81310, Malaysia (Corresponding author: Shaharil Mad Saad; e-mail: shaharil@utm.my). technical application for example guiding drones through in door environments or locating cars inside a tunnel [4].

In this paper, GUI based on Labview software which is used to determine position is developed. GUI in term of graphical, it has tools bar in block diagram that can be used friendly for writing a coding and run a program. This paper is organized as follows: Local Positioning System in Section II. LPS Architecture in Section III. Triangulation Method is introduced in Section IV. Graphical User Interface and experiment test are presented in section V and Section VI, respectively. The test verification is introduced in Section VII. Finally, conclusion is drawn in Section VIII.

\section{LOCAL POSITIONING SYSTEM (LPS)}

LPS as show in Fig. 1 starts with the arrangement of three anchors and one tag for getting tag's positioning in term of $(\mathrm{X}, \mathrm{Y})$ direction. Attached with the anchors is future technology device international (FTDI). It is used for firmware uploading from computer. The LPS also consist of a router. It will send data that contain all information about each anchor to computer. The technology used for this LPS is UWB based on triangulation method and time different of arrival (TDOA) technique. There are four different techniques used in previous literature for finding the position in LPS which are: Time of Arrival (TOA), Time Different of Arrivals (TDOA), Angel of Arrival (AOA) and Received Signal Strength Indicator (RSSI) [7]. This study used TDOA technique since it does not need to be synchronized between a transmitter and receiver and synchronize only between receivers. However, the time of receiver section it needs to be synchronized at different receiver sections measurement, receivers must be paired to get TDOA measurements to any location which want to be estimated [8], [9]. TDOA measurements computes from the received signal for at least three receivers to provide the transmitter position [10]-[12]. This computes as follow [12]:

$$
\Delta d=c^{*} \Delta t
$$

where,

$c$ represent speed of light, $\Delta \mathrm{t}$ is deferent time of arrival at each receiver. Thus, in term of coordinate the difference, $\Delta d$ can be written as follow:

$$
\Delta d=\sqrt{\left(x_{2}-x\right)^{2}-\left(y_{2}-y\right)^{2}}-\sqrt{\left(x_{1}-x\right)^{2}-\left(y_{1}-y\right)^{2}}
$$

where: $\left(x_{1}, y_{1}\right)$ represent position of the receiver 1 and $\left(x_{2}, y_{2}\right)$ represent position of the receiver 2,(both are known position $).(x, y)$ is unknown position of source position. 
UWB can be defined as a wireless technology. It uses ultra -short pulse [13] to calculate positioning based on radio signals which are traveling receiver node target and transmitter node which is has known position. It provides a higher accuracy, good precise distance measurement and coverage area is $15-25 \mathrm{~m}$ in 2-D area [14]. It has low complexity, both of security and scalable are high, low power consumption and high data rate transmission. It has ability to resistance to multi path effects [10], [15]. Finally, the cost is cheaper. Therefore, UWB becomes attractive in different applications like tracking [16]. UWB operates by transmitting and receiving an exceptionally short duration burst of RF energy-typically a few tenths of a picosecond to a few nanoseconds in duration [17]. By utilizing both of technology (UWB) and technique (TDOA) which is chosen to provide good accuracy for LPS.

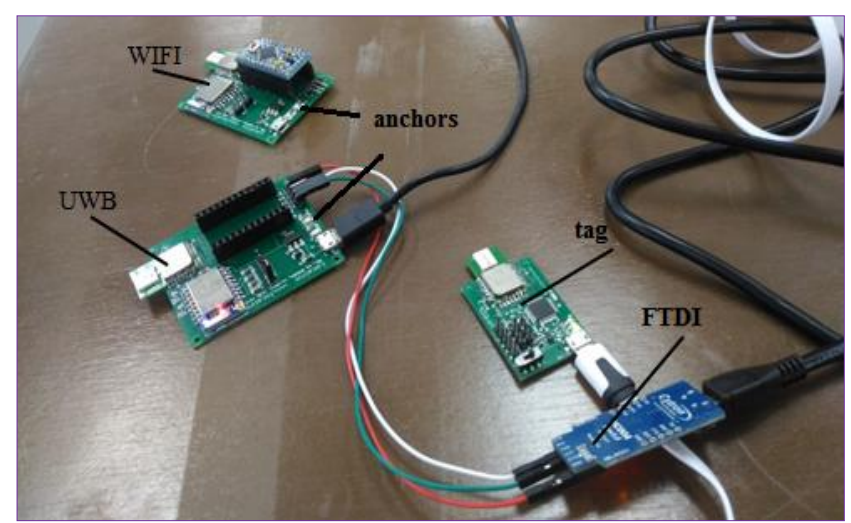

Fig. 1. LPS's hardware.

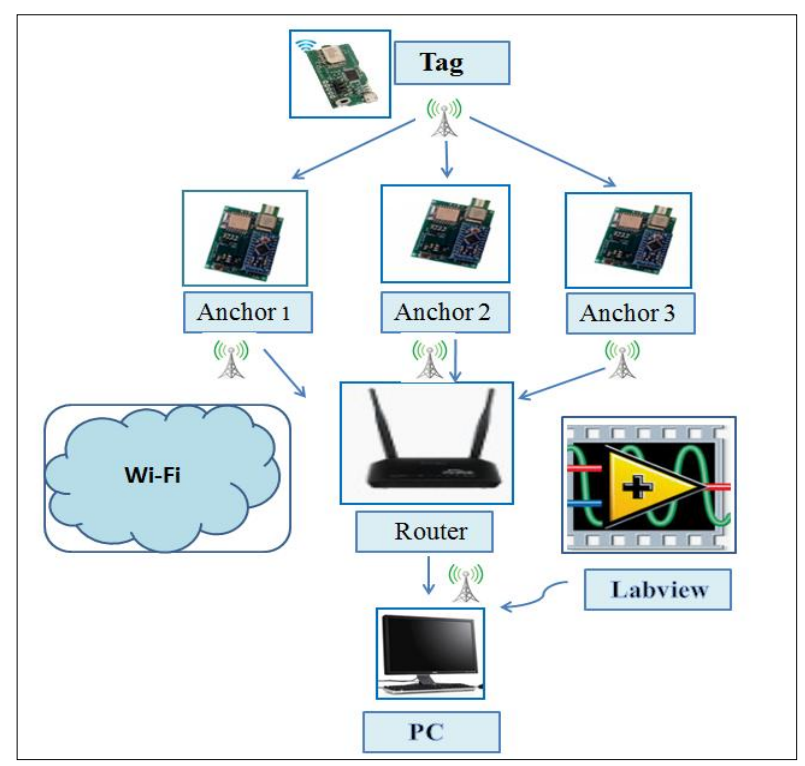

Fig. 2. LPS architecture.

\section{LPS ARCHITECTURE}

In LPS, three anchors at least with known position are employed with one tag to get the position $(x, y)$. Fig. 2 shows the LPS architecture where the tag sends signal through UWB to three anchors. The anchors received the signal and sent it to a router. This router contain all information about the electric devices (anchors) which is used in LPS such as (IP address for each one, and special name like AA: 01, refer to anchor 1; $\mathrm{AB}: 02$, refer to anchor 2; $\mathrm{AA}: 03$, refer to anchor
3. Then the router will send data depending on triangulation method to PC. Software processor using labview environment The Environment in Labview is composed of two part. One is a front panel that considered as the interface with all the controls and final results. Second part is block diagram where the actual flow of operations occurs base on function blocks with inputs and outputs. The conjunction of the front panel and the block diagram form what is called a virtual instrument (VIs).

\section{TRiangulation Method}

The triangulation method used trigonometry to determine the position of objects [18]. It used to calculate the positioning of a point by measuring angles from this point at each end of fixed baseline instead of estimated distance to the point directly [19]. The main idea of triangulation method is to estimate distance between tag that emitting signals to all anchors at synchronize time [20].

In 2D environment, at least three anchors and one tag are needed. The triangulation method is based on the known radius $r_{1}, r_{2}$, and $r_{3}$ which is shown in Fig. 3 .

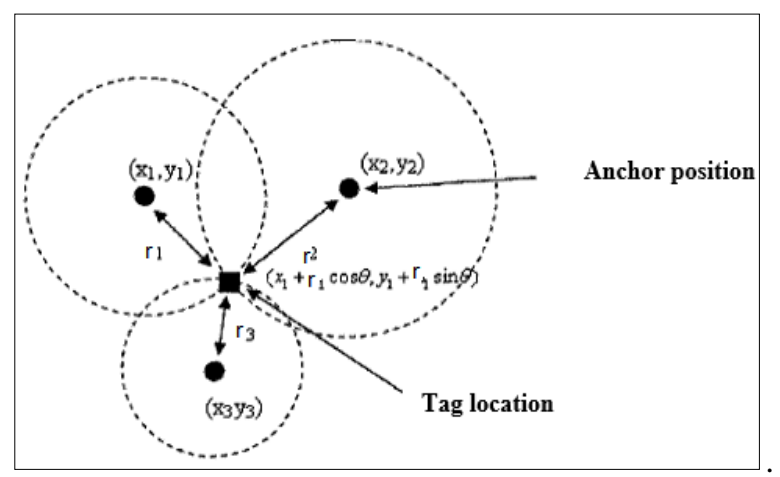

Fig. 3. Triangulation method.

By applying theorem Pythagoras, those radius are calculated as shown in following equations.

$$
\begin{aligned}
& \left(x-x_{1}\right)^{2}+\left(y-y_{1}\right)^{2}=r_{1}{ }^{2} \\
& \left(x-x_{2}\right)^{2}+\left(y-y_{2}\right)^{2}=r_{2}{ }^{2} \\
& \left(x-x_{3}\right)^{2}+\left(y-y_{3}\right)^{2}=r_{3}{ }^{2}
\end{aligned}
$$

By solving those equations, the tag's positioning $(x, y)$ can be determined by following equations:

$$
\begin{gathered}
X=\frac{\mathrm{FB}-\mathrm{CE}}{\mathrm{DB}-\mathrm{AE}} \\
Y=\frac{\mathrm{DC}-\mathrm{AF}}{\mathrm{DB}-\mathrm{AE}}
\end{gathered}
$$

where:

$$
\begin{gathered}
\mathrm{A}=\left(2 x_{2}-2 x_{1}\right) \\
\mathrm{B}=\left(2 y_{2}-2 y_{1}\right) \\
\mathrm{C}=r_{1}{ }^{2}+x_{2}{ }^{2}+\mathrm{y}_{2}{ }^{2}-\mathrm{r}_{2}{ }^{2}-\mathrm{x}_{1}{ }^{2}-\mathrm{y}_{1}{ }^{2} \\
\mathrm{D}=\left(2 x_{3}-2 x_{2}\right) \\
\mathrm{E}=\left(2 y_{3}-2 y_{2}\right) \\
\mathrm{F}=r_{2}{ }^{2}+{x_{3}}^{2}+{y_{3}}^{2}-{r_{3}}^{2}-x_{2}{ }^{2}-y_{2}{ }^{2}
\end{gathered}
$$


Another way to get position $(x, y)$, instead of by knowing the radius is based on angles $(\alpha, \beta)$ and baseline, $\mathrm{AB}$ method as shown in Fig. 4.

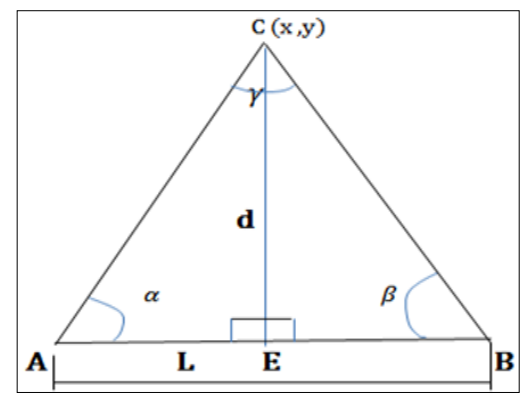

Fig. 4. Concept of triangulation method

The position $(x, y)$ can be determined by using (8) and (9).

$$
\begin{aligned}
& \left(x-x_{1}\right)^{2}+\left(y-y_{1}\right)^{2}=A C^{2} \\
& \left(x-x_{2}\right)^{2}+\left(y-y_{2}\right)^{2}=B C^{2}
\end{aligned}
$$

where:

$$
\begin{array}{r}
\mathrm{AC}^{2}=\mathrm{AE}^{2}+d^{2} \\
\mathrm{CB}^{2}=\mathrm{BE}^{2}+d^{2} \\
d=L\left(\frac{\sin \alpha \sin \beta}{\sin (\alpha+\beta)}\right) \\
\mathrm{AE}=\frac{d}{\tan \alpha} \\
\mathrm{EB}=\frac{d}{\tan \beta}
\end{array}
$$

\section{GRAPHICAL USER INTERFACE (GUI)}

In this paper, the GUI was developed based on Labview environment. It is used to determine tag's position. GUI is a communication tools which is easier to link both of user and system and can creating interface path between them [21]. It should be developed so it can use for multi-purpose like inputs and also utilize to display the result [22]. In order the GUI is able to work properly in a given task, it needs to have a few common functions. The function can be added into the GUI for processing and visualize any measurement data [23]. Basically, Labview is a platform that uses visual programming language from National Instruments (NI). It is developed by National Instruments to help us to make good interface between the PC and the electric device. It works very well with data communication such as user datagram protocol (UDP) and Transmission Control Protocol/Internet Protocol (TCP/IP). Finally, it is easy-to-use graphical interface compatible with the electric devices to configure it and run it with very few built-in block diagram [24].

Fig. 5a shows the front panel for the GUI that has been developed by using Labview software for determine the tag's positioning. The front panel consist of file's path location for data recorded. It also shows the IP address for each anchor will check in Labview software by using data communication in NI where positioning for each anchor is checked separated where receiving the data. All information such as name and radius for each anchors are displayed. Figure.5b shows the block diagram where the coding is written based on Labview software by using UDP and TCP/ IP communication tool. lastly IP address for LPS will recognize and read depending on UDP tool which is appearance in Front panel of GUI. Data for each anchors will update and all data will save in specific file in Labview folder. The GUI also can calculate the average accuracy between real position and the estimated position which is to be targeted less than $17 \mathrm{~cm}$. It regards as a good accuracy for this kind of LPS.

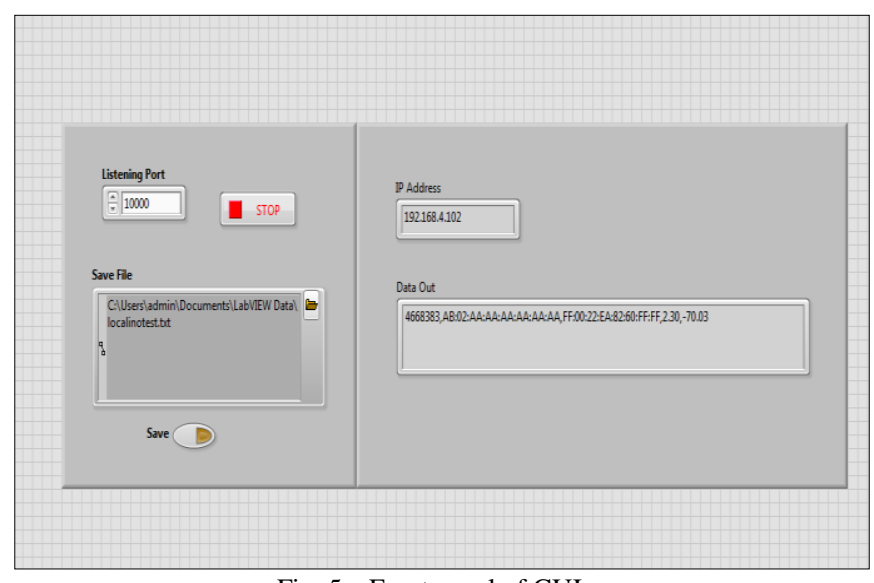

Fig. 5a. Front panel of GUI.

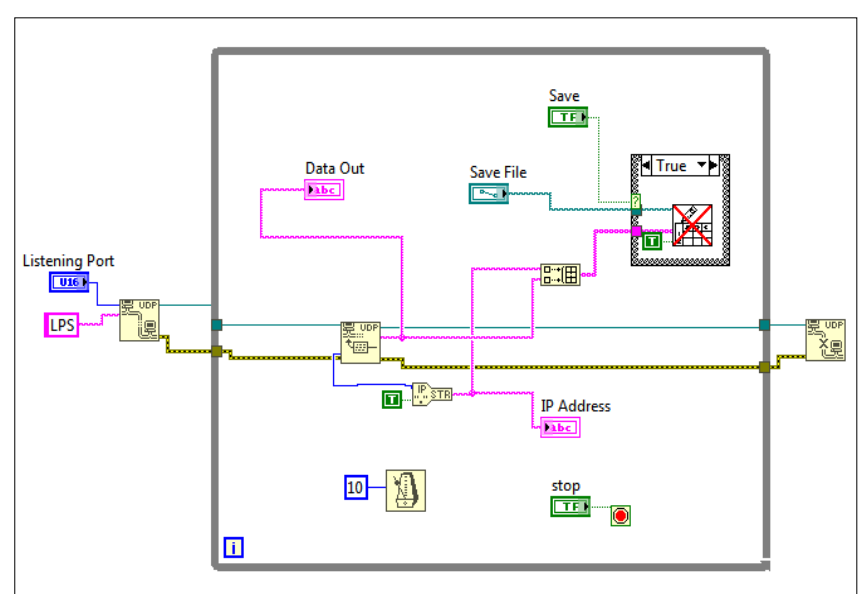

Fig. 5b. Block diagram of GUI.

\section{EXPERIMENT TEST}

An experiment has been developed to test the functioning of GUI. The demonstrated system comprise of four electrical devices : anchor 1, anchor 2, anchor 3 and one tag. The tag transmits UWB pulses continuously to the anchors. In each measurement series reads UWB signals emitted from the tag more several times. Each measured with range (r) for three anchors $\mathrm{AA}=01, \mathrm{AB}=02, \mathrm{AA}=03$. Positioning error between true position and the estimated position is about $17 \mathrm{~cm}$. In Fig. 6 , three anchors are set at the specific (known) position in $(x$, $y$ ) direction which is anchor $1(0,0)$, anchor $2(2,0)$ and anchor 3 (2,2). Unit of coordination system is in meter. Three anchors are setup in three corners of a squared $2 \mathrm{~m} \times 2 \mathrm{~m}$ area, as depicted below.

The origin of coordinate system is set at one of the corner of the squared in $(0,0)$ area. As it is known the geometric placement of the anchors directly determines the dilution of precision (DOP) that measures sensitivity of result which appears against error at input [25], anchors are placed at 
height $143 \mathrm{~cm}$ to achieve lowest DOP as shown in Fig. 7. In fact, any obstacle near the anchors location should be taken into account. It may degrade the accuracy. System performance reported in this work was based on measurement 16 positions distributed within the area $2 \mathrm{~m} \times 2$ m. For getting the accuracy, the tag will move at various chosen points within the square area.

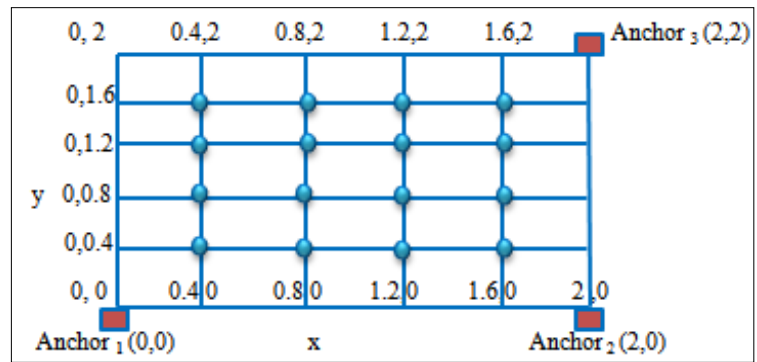

Fig. 6. Anchors location.

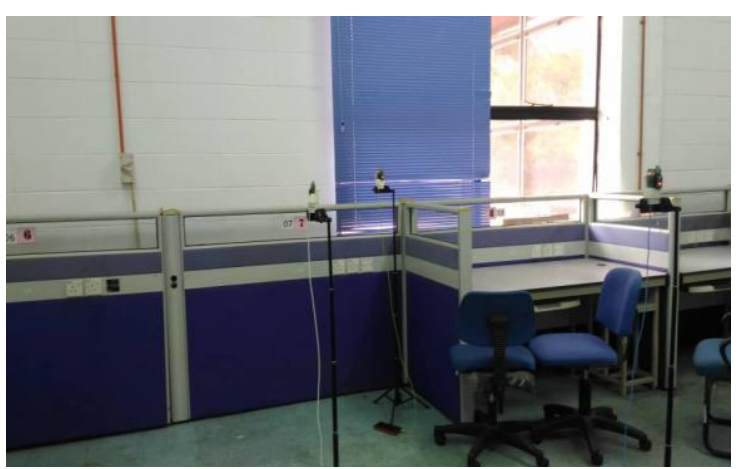

Fig. 7. Tag and anchors positioning configuration in lab.

\section{RESUlT AND DISCUSSION}

A test has been done to determine local positioning that using three anchors and one tag. All anchors are arranged in a fixed position and tag is moved at different location. Data received from each anchors is presented in the table of the GUI front panel. The result is shown in Fig. 8a and Fig. 8b at different of Tag's position. The result of tag's position is plotted in an $x, y$ graphical graph. The Tag's positioning in a point $(x, y)$ will be updated immediately after changing it is location. The GUI also presented information for each anchor like IP address and data header in real time.

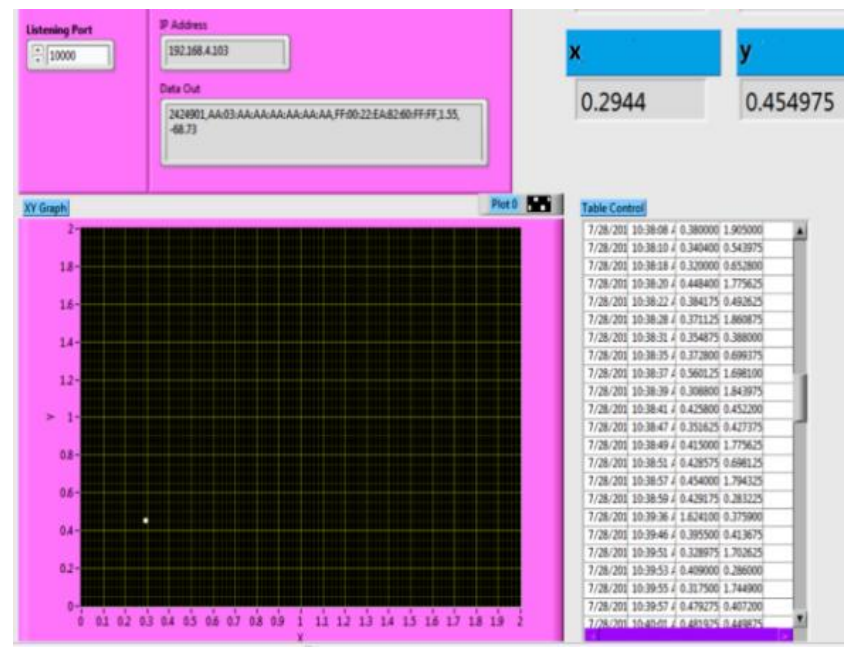

Fig. 8a. Result of tag's position1.

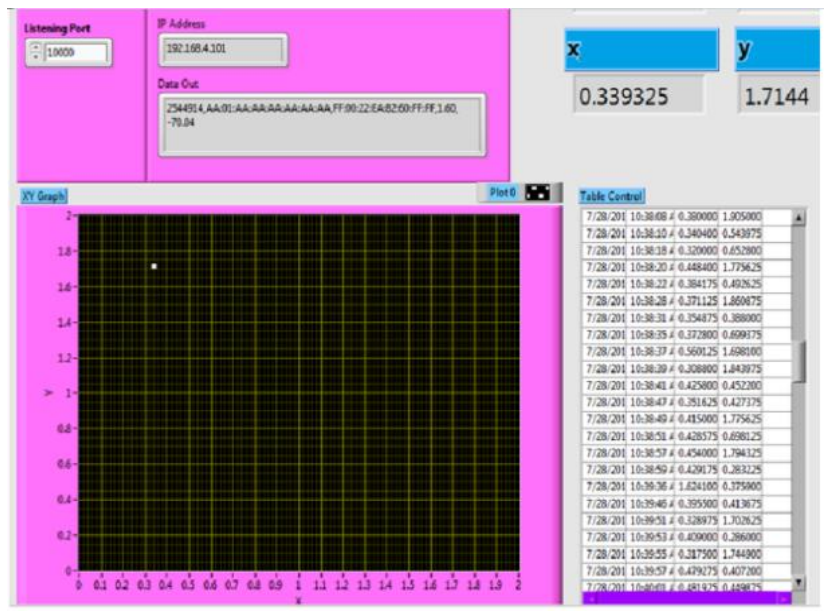

Fig. 8b. Result of tag's position 2 .

Fig. 9a shows an average value displayed on Labview front panel for $x$ and $y$ direction while the Fig. 9b shows it's block diagram of GUI. It shows the contain of total measurement or samples that are used in LPS experiment. Each measurement in $(x, y)$ direction takes for 100 sample in the same position which is tag's position to get better accuracy. By using GUI the accuracy of LPS based on labview environment is achieved to provide good accuracy value.

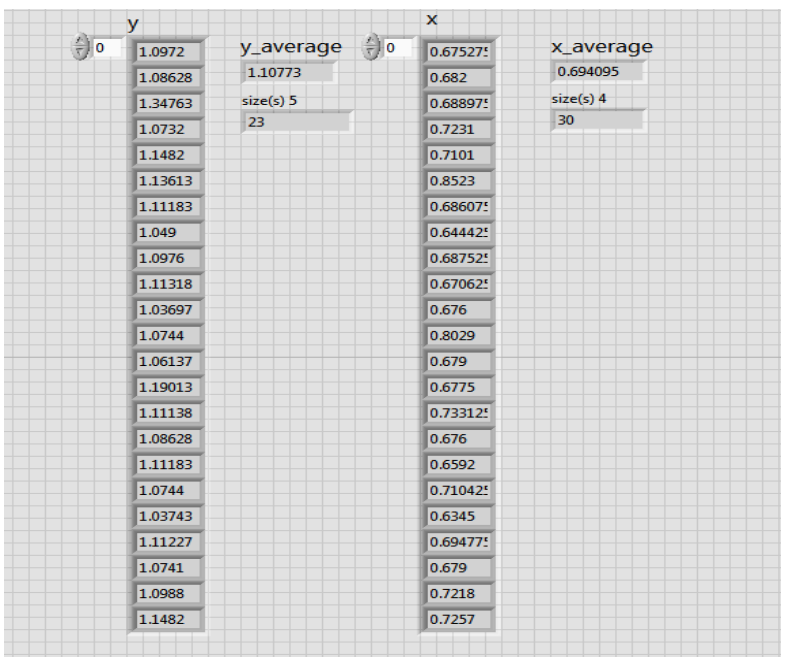

Fig. 9a. Front panel of GUI.

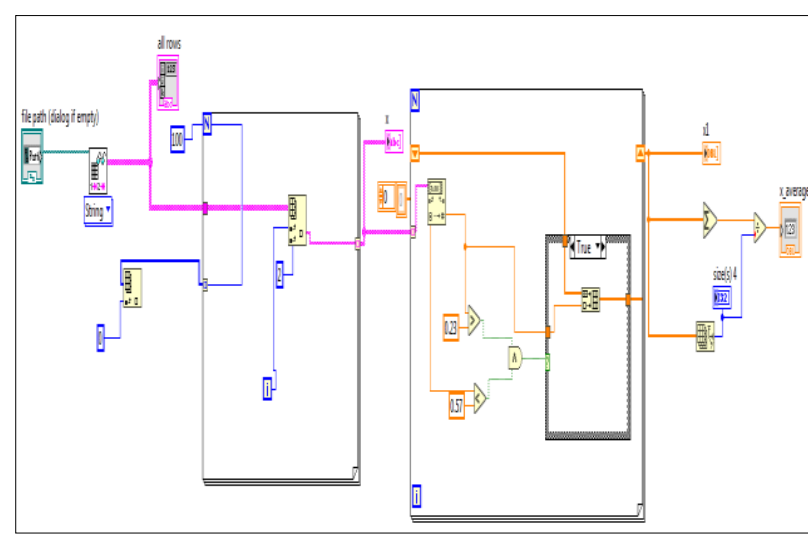

Fig. 9b. Block diagram of GUI

Table I shows the real and average measured value from GUI for 100 samples. It shows that the accuracy of $x$ direction and $y$ direction. The maximum value for the accuracy of $x$ and $y$ direction is $16.8 \mathrm{~cm}$ and $15 \mathrm{~cm}$ respectively. While the 
minimum value is $0.3 \mathrm{~cm}$ and $1.2 \mathrm{~cm}$ for $x$ and $y$. Depending on the triangulation method the best accuracy can be achieved in center of selected area that closed to its real value. This shows that the accuracy are less than $17 \mathrm{~cm}$.

Fig. 10 illustrates a graph that presented the point $(x, y)$ for real and measured values. The measured value is an average of 100 samples at different Tag position in $(x, y)$ direction. It shows that the measured values are near to its real values.

TABLE I: REAL AND MEASURED VALUE

\begin{tabular}{|c|c|c|c|c|c|c|}
\hline \multirow[t]{2}{*}{ No } & \multicolumn{2}{|l|}{$\begin{array}{l}\text { Real } \\
(\mathbf{c m})\end{array}$} & \multicolumn{2}{|c|}{$\begin{array}{l}\text { Average measured } \\
\text { values }(\mathrm{cm})\end{array}$} & \multicolumn{2}{|c|}{ Accuracy $(\mathrm{cm})$} \\
\hline & $\mathbf{x}$ & $\mathbf{y}$ & $\mathbf{x}$ & $\mathbf{y}$ & $\mathbf{x}$ & $\mathbf{y}$ \\
\hline 1 & 40 & 80 & 29.3 & 72.4 & 10.7 & 7.6 \\
\hline 2 & 40 & 120 & 45.3 & 110.7 & 5.3 & 9.3 \\
\hline 3 & 40 & 160 & 39.7 & 173 & 0.3 & 13 \\
\hline 4 & 80 & 80 & 68.6 & 75.2 & 11.4 & 4.8 \\
\hline 5 & 80 & 120 & 69.1 & 118.8 & 10.9 & 1.2 \\
\hline 6 & 80 & 160 & 71.7 & 172.7 & 8.3 & 12.7 \\
\hline 7 & 120 & 80 & 116.8 & 67.9 & 3.2 & 12.1 \\
\hline 8 & 120 & 120 & 109.9 & 127.1 & 11.1 & 7.1 \\
\hline 9 & 120 & 160 & 113.8 & 166.7 & 6.2 & 6.7 \\
\hline 10 & 160 & 40 & 152.7 & 38 & 7.3 & 2 \\
\hline 11 & 160 & 80 & 143.2 & 70 & 16.8 & 10 \\
\hline 12 & 160 & 160 & 154 & 175 & 6 & 15 \\
\hline
\end{tabular}

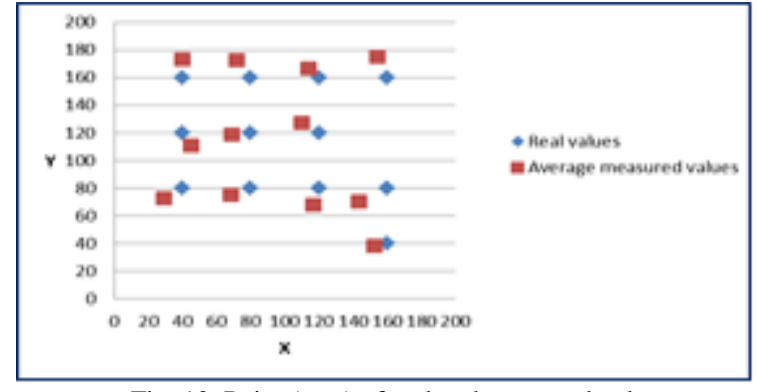

Fig. 10. Point $(x, y)$ of real and measured values.

\section{CONCLUSION}

In this paper, the GUI for LPS that consists of three anchors and one tag has been successfully developed by using Labview software. This software is from National Instruments (NI Labview). It has been chosen because it works very well with many functions such data communication UDP and TCP/IP, etc. IP address and data for each anchor is read by using Labview data communication tools. The data received from three anchors are recorded and analyzed based on TDOA technique. The result shows that the GUI can updates the tag's positioning immediately when its location is changed from time to time. An experiment also has been conducted for test the functioning of GUI where 100 data samples are collected. The result shows that, the GUI is work properly where the data received and analyzed from GUI can give the tag's positioning accuracy less than $17 \mathrm{~cm}$. It is a good accuracy for LPS.

\section{ACKNOWLEDGEMENT}

The authors wish to thank Universiti Teknologi Malaysia
(UTM) for providing the funding and facilities to conduct this research with Project No. Q.J130000.2524.20H25.

\section{REFERENCES}

[1] J. D. Domingo et al., "Indoor positioning system using depth maps and wireless networks," Journal of Sensors, pp. 1-8, 2016.

[2] C. Basri and A. E. Khadimi, "Survey on indoor localization system and recent advances of WIFI fingerprinting technique," in Proc. International Conference on Multimedia Computing and Systems, 2017, pp. 253-259.

[3] Z. Farid, R. Nordin, and M. Ismail, "Recent advances in wireless indoor localization techniques and system," Journal of Computer Networks and Communications, pp. 1-12, 2013.

[4] R. Khullar and Z. Dong, "Indoor localization framework with WiFi fingerprinting," in Proc. 2017 26th Wireless and Optical Communication Conference (WOCC) USA, 2017, pp. 1-6.

[5] R. F. Brena et al., "Evolution of indoor positioning technologies: A survey," Journal of Sensors, 2017

[6] L. Mainetti, L. Patrono, and I. Sergi, "A survey on indoor positioning systems," in Proc. 2014 22nd International Conference on Software, Telecommunications and Computer Networks, 2014, pp. 111-120.

[7] H. Sahib and S. M. S. M. Hussein, "An overview of local positioning system technologies, techniques and applications," International Journal of Engineering and Technology, vol. 7, no. 3, pp. 1-5, August, 2018.

[8] M. Vossiek et al., "Wireless local positioning," IEEE Microwave Magazine, pp. 77-86, December, 2003.

[9] K. . O. Brian, Finding Location with Time of Arrival and Time Difference of Arrival Techniques, pp. 1-3, 2017.

[10] K. Mannay et al., "Location and positioning systems: Performance and comparison," in Proc. 2016 4th International Conference on Control Engineering and Information Technology (CEIT), December 2016, pp. 1-6.

[11] J. Miši, "An overview of wireless indoor positioning systems," Infoteh-Jahorina, vol. 14. pp. 301-306, March 2015.

[12] S. Link, "Positioning using time-difference of arrival measurements fredrik gustafsson and fredrik gunnarsson department of electrical engineering," Electrical Engineering, pp. 8-11, 2013.

[13] L. Angrisani, P. Arpaia, and D. Gatti, "Analysis of localization technologies for indoor environment," in Proc. 2017 IEEE International Workshop on Measurement and Networking, 2017.

[14] A. Yassin et al., "Recent advances in indoor localization: A survey on theoretical approaches and applications," IEEE Communications Surveys and Tutorials, vol. 19, no. 2, pp. 1327-1346. November, 2016.

[15] A. Ren et al.,"A study of indoor positioning based on UWB base-station configurations," in Proc. 2017 IEEE 2nd Advanced Information Technology, Electronic and Automation Control Conference (IAEAC), 2017, pp. 1939-1943.

[16] S. Hu, M. Kang, and C. She, "Vehicle positioning based on UWB technology," Journal of Physics: Conference Series, 2017.

[17] Mikhaylov et al., "On the selection of protocol and parameters for UWB-based wireless indoors localization," in Proc. International Symposium on Medical Information and Communication Technology, 2016.

[18] E. C. C. Lun, "An accurate and robust indoor localization system," Doctoral Dissertation, Hong Kong Polytechnic University, China, p. 196, 2010 .

[19] O. Motlagh et al., "A review on positioning techniques and technologies: A novel AI approach,', Journal of Applied Sciences, pp. 1601-1614, 2009.

[20] S. Ravindra and S. N. Jagadeesha, "Time of arrival based localization in wireless sensor networks: A linear approach," Signal and Image Processing: An International Journal, vol. 4, no. 4, pp. 13-30, 2013.

[21] S. Sulaiman et al., "Graphical user interface ( GUI ) development for object tracking system in video sequences," World Applied Sciences Journal , vol. 4, no. 2, pp. 244-249, 2008.

[22] C. Y. Huang, L. M. Liu, and Y. L. Chen, "An online integrated fingerprint image system," International Journal of Machine Learning and Computing, vol. 9, no. 1, pp. 51-56, February 2019.

[23] F. Mochammad, A. R. Putra, and B. R. Trilaksono, "Implementation of hazardous chemical gas monitoring system using unmanned aerial vehicle ( UAV ), ” pp. 163-168, 2016.

[24] National instruments, Inc. (March, 2015). What Is Labview? [Online] Available: http://www.ni.com/newsletter/51141.

[25] H. T. Dinh, C. L. Law, and R. C. L. Wei, "3-D localization system using 4 anchors employing ultra-wideband impulse radio," in Proc. 2015 10th International Conference on Information, Communications and Signal Processing, 2015, vol. 2, pp. 1-5. 


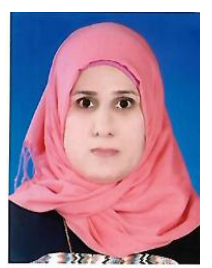

Hameedah Sahib Hasan received her B.E. in mechanical department from Technology University Bagdad, Iraq; A master engineering in mechanical engineering, automation and robotics from University College of Engineering, Osmania University, Hyderabad, India, 2013. Currently, She is working towards a doctoral degree from School of Mechanical, Faculty of Engineering, Universiti Teknologi, Malaysia. At present she is working as an assistant lecturer, in Ministry of Higher Education and Scientific Research, Al-Furat Al-Awsat Technical University, Iraq.

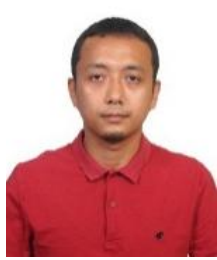

Mohamed Hussein received his B.E. in mechanica engineering from Universiti Teknologi, Malaysia, master engineering in advanced manufacturing system and technology from University of Liverpool, UK 1992 and $\mathrm{PhD}$ in mechatronics from De Montfort University, UK, 2008. His research interest includes intelligent control and automation, mechatronics, robotics. He has published more than 40 internationa conference and 30 journal papers. He has 3 Book Chapters, 10 Awarded Projects and 8 Patent Filing. Currently he is work as associate professor in the Faculty of Mechanical Engineering, UTM Malaysia.

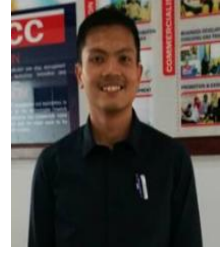

Shaharil Mad Saad was born in 1985 . He received his B.E in electrical engineering, instrumentation \& control from Universiti Teknologi Malaysia (UTM) in 2008; a master engineering in mechanical engineering from University Teknologi, Malaysia, 2011 and a $\mathrm{PhD}$ in mechatronic engineering from Universiti Malaysia Perlis (UniMAP), Malaysia in 2016. His reseach interest includes embedded system, control and instrumentation system, artificial intelligence. He has published more than 16 international conference and 11 journal papers. He has 4 awarded projects. Currently he is work as a senior lecturer in the Faculty of Mechanical Engineering, UTM, Malaysia.

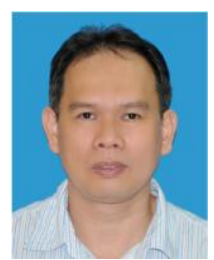

Mohd Azuwan Bin Mat Dzahir received his B.E. in mechanical engineering from University Teknologi, Malaysia in 2009; a master engineering in mechanical engineering from University Teknologi, Malaysia in 2010, and $\mathrm{PhD}$ in functional control system and a sub-major in business development from Shibaura Institute of Technology (SIT), Japan in 2014. His research interest includes rehabilitation robotics, gait and posture, intelligent control and automation, hysteresis modeling. Currently he is work as a senior lecturer in the Faculty of Mechanical Engineering, UTM, Malaysia. 